\title{
Editorial
}

\section{Five years of percutaneous transluminal septal myocardial ablation}

In June 1994, Ulrich Sigwart injected a small quantity of absolute alcohol into the first septal artery of a 67 year old woman with hypertrophic obstructive cardiomyopathy. ${ }^{1}$ The objective of this somewhat alarming manoeuvre was to produce an area of localised myocardial infarction in the left ventricular outflow tract-in effect producing a chemical myotomy-myectomy, without recourse to open heart surgery. Five years on, the technique has been applied by a number of groups in a few hundred patients worldwide.

The birth of the Sigwart procedure (percutaneous transluminal septal myocardial ablation, transcoronary ablation of septal hypertrophy, non-surgical septal reduction) was not greeted with unanimous approval from the community of physicians treating patients with this troublesome condition. A number of eminent commentators have referred to the procedure's "profoundly aggressive" nature and its associated "unacceptably high mortality and morbidity". ${ }^{2}$ Clearly such comments were made at a very early point in the technique's development; it is now five years old, what do we know of the results of this interventional "enfant terrible" and how safe is it?

Information is still restricted to data from relatively few patients with (inevitably) only medium term follow up. There have been five reports of the short term results of the procedure involving just under 200 patients, ${ }^{3-7}$ and three longer term (7-30 months) reports ${ }^{7-9}$ in fewer than 100 patients - one of which (Faber et al) is published in this issue of Heart. ${ }^{8}$ Clearly such limited information does not allow an equitable comparison to be made with surgical myotomy-myectomy, but should be enough to distinguish a promising treatment from a potential disaster.

\section{Mortality}

The overall published procedural mortality is $2 \%$ (four deaths)..$^{3-7}$ Two of the deaths occurred in patients with severe co-existent pulmonary disease, one occurred as the result of pulmonary embolism (probably as a result of femoral vein thrombosis associated with a temporary pacing wire), and one was the consequence of sudden onset atrioventricular (AV) block four days after the procedure. Over the longer term there has been only one further death reported (from pancreatic carcinoma). ${ }^{9}$ The wisdom of generalising from this preliminary mortality rate is debatable. The reported series represent the steepest portion of the learning curve and initially one group restricted the procedure to patients with "severe co-morbidity". ${ }^{7}$ However, the reports originate from centres with the greatest expertise in performing septal ablation, and results in less experienced hands may be worse. This situation may mirror the results of surgery, where mortality after myotomy-myectomy in selected patients in experienced centres is less than $2 \%,{ }^{10}$ but is increased in high risk subgroups $(18 \%)^{11}$ or in smaller volume units.

\section{Complications}

The main complication of the Sigwart procedure is the induction of $\mathrm{AV}$ block requiring permanent pacemaker implantation. The overall requirement for pacing is $21 \%$, varying from $0-40 \%$ among reports. ${ }^{3-7}$ The reason for this wide variation in pacing rates is still unclear but, anecdotally, most centres are reporting a reduction in the need for pacing, perhaps as a result of improved targeting of the ablation site with myocardial contrast echocardiography. ${ }^{6}$ It does not seen unreasonable to suppose that the rate of pacemaker implantation after the Sigwart procedure will become broadly similar to that seen after myotomymyectomy (around 10\%). ${ }^{12}$

The deliberate creation of a myocardial infarct may create a long term substrate for arrhythmias and promote adverse ventricular remodelling. Consequently, most investigators provide data on ventricular dysrhythmias and left ventricular cavity size. Early ventricular arrhythmias occur in fewer than $2 \%$ of patients, usually without significant sequelae. There have been no reports of later onset ventricular arrhythmias to date. Faber et al report no significant change in left ventricular end diastolic diameter over 30 months' follow up, ${ }^{8}$ but another longer term report ${ }^{9}$ did detect a small $(4 \mathrm{~mm})$ but significant increase in left ventricular end diastolic diameter with time. While encouraging, the follow up intervals of these reports are too brief to provide complete reassurance that the beneficial early haemodynamic effects of the Sigwart procedure are not bought at the expense of longer term ventricular function.

\section{Effectiveness}

There seems little doubt that the Sigwart procedure has equivalent efficacy to surgical techniques in the reduction of left ventricular outflow tract obstruction. Pooled data ${ }^{3-7}$ suggest a reduction in gradient from a mean of $60 \mathrm{~mm} \mathrm{Hg}$ to $14 \mathrm{~mm} \mathrm{Hg}$, and studies with longer follow up ${ }^{7-9}$ do not show any recurrence of obstruction with time.

Symptomatic rather than haemodynamic changes following a new interventional procedure are extremely difficult to assess as a consequence of a substantial placebo effect, and neither surgery nor the Sigwart procedure can be subjected to the rigorous double blind evaluation that has diminished enthusiasm for dual chamber pacemaker treatment for hypertrophic cardiomyopathy. ${ }^{13}$ Bearing these important caveats in mind, all three longer term reports show significant and maintained improvements in symptoms with a mean increase of just over one NYHA (New York Heart Association) functional class. ${ }^{7-9}$ More importantly, objective tests of exercise capacity show increases of around $40 \%$ in exercise performance over follow up. ${ }^{78}$

We are now at the stage when the Sigwart procedure should be compared with surgical myotomy-myectomy in a randomised trial. This will not be an easy undertaking. First, there may be a tendency for patients to opt for the less aggressive technique rather than randomisation, arguing (not unreasonably) that surgery can be performed if ablation fails. Second, the number of patients for whom either technique is appropriate is small. The success of the technique in the future depends critically on correct patient selection. Left ventricular outflow tract obstruction in hypertrophic cardiomyopathy is compatible with a normal life expectancy in some patients and there is no evidence that relief of obstruction has an impact on prognosis. It follows that myotomy-myectomy or the Sigwart procedure should be performed only on patients 
with severe symptoms that have not responded to conventional medical treatment and who have significant obstruction ( $\geqslant 50 \mathrm{~mm} \mathrm{Hg}$ at rest). The procedure should be performed by individuals with experience of the technique, supported by a department with an interest in the disease either as part of a randomised trial or at least reporting to a centralised registry. There is a danger that the elegance and simplicity of the Sigwart procedure may lead interventional cardiologists, whose expertise lies primarily in coronary angioplasty, to underestimate the difficulties of provoking therapeutic myocardial infarction in patients with hypertrophic cardiomyopathy. If performed on an ad hoc basis in poorly selected patients the reputation of the Sigwart procedure will be irreparably damaged. This would be a pity as the signs from these early studies are that the procedure may be as effective and as safe as surgery, giving patients and physicians a choice, where none before existed. If carefully nurtured, the Sigwart procedure, like so many enfant terribles, may enjoy a rather respectable middle age.

London Chest Hospital, Bonner Road, London E2 97X, UK

CHARLES J KNIGHT

1 Sigwart U. Non-surgical myocardial reduction for hypertrophic obstructive cardiomyopathy. Lancet 1995;346:211-14.

2 Maron BJ, Spirito P, McKenna WJ, et al. The management of hypertrophic cardiomyopathy [letter]. N Engl f Med 1997;337:349-50.
3 Knight C, Kurbaan AS, Seggewiss H, et al. Nonsurgical septal reduction for hypertrophic obstructive cardiomyopathy. Outcome in the first series of patients. Circulation 1997;95:2075-81.

4 Seggewiss H, Gleichmann U, Faber L, et al. Percutaneous transluminal septal myocardial ablation in hypertrophic obstructive cardiomyopathy: acute results and three-month follow-up in 25 patients. F Am Coll Cardiol 1998; 31:252-8.

5 Faber L, Seggewiss H, Gleichmann U. Percutaneous transluminal septal myocardial ablation in hypertrophic obstructive cardiomyopathy. Results with respect to intraprocedural contrast echocardiography. Circulation 1998;98:2415-21.

6 Lakkis NM, Nagueh SF, Kleiman NS, et al. Echocardiography-guided ethanol septal reduction for hypertrophic obstructive cardiomyopathy. Circulation 1998;98:1750-5.

7 Gietzen FH, Leuner CJ, Raute-Kreinsen U, et al. Acute and long term results after transcoronary ablation of septal hypertrophy (TASH). Catheter interventional treatment for hypertrophic cardiomyopathy. Eur Heart $\mathcal{F}$ 1999;20:1342-54.

8 Faber L, Meissner A, Ziemssen P, et al. Percutaneous transluminal septal myocardial ablation for hypertrophic obstructive cardiomyopathy: long term follow up of the first series of 25 patients. Heart 2000;83: $326-31$

9 Harrington D, Shamim W, Knight C, et al. Long term follow-up of hypertrophic myopathy patients treated with non-surgical septal reduction [abstract]. Heart 1999;81(suppl 1):P5.

10 Maron BJ. Hypertrophic cardiomyopathy. Lancet 1997;350:127-33.

11 Siegman IL, Maron BJ, Permutt LC, et al. Results of operation for coexistent obstructive hypertrophic cardiomyopathy and coronary artery disease. f Am Coll Cardiol 1989;13:1527-33.

12 Heric B, Lytle BW, Miller DP, et al. Surgical management of hypertrophic obstructive cardiomyopathy. Early and late results. $\mathcal{F}$ Thorac Cardiovasc Surg 1995;110:195-208.

13 Nishimura RA, Trusty JM, Hayes DL, et al. Dual-chamber pacing for hypertrophic obstructive cardiomyopathy: a randomised double-blind crossover study. $\mathcal{F}$ Am Coll Cardiol 1997;29:1039-45.

\section{STAMPS IN CARDIOLOGY}

\section{Acupuncture anaesthesia for open heart surgery}

Open heart surgery under acupuncture anaesthesia is depicted on this 8 cent Chinese stamp issued in 1975 as part of a set of four stamps to commemorate the successful integration of traditional Chinese medicine and modern Western medicine in the treatment of various diseases. The four Chinese characters at right lower corner of the stamp stand for acupuncture anaesthesia. The other three stamps in the set feature such surgical feats as replantation of severed limbs, application of small soft splints for fractures, and cataract surgery.

The patient in this stamp was a 15 year old girl with congenital ventricular septal defect. The Chinese made disc oxygenator for total cardiopulmonary bypass can be seen on the right. The anaesthetist who performed the acupuncture - two fine needles inserted in both wrists and a further two in the anterior chest wall at both subclavicular areas-was at the patient's head; he was a practitioner of Chinese traditional medicine. The surgeon on the patient's left was Professor Yi-shan Wang, my schoolmate in St John's University School of Medicine, Shanghhai, China, and the surgeon on the patient's right was Dr Chun-xiu Yeh, my classmate in the same school.

From the historical record of Huangdi Neijin (The Yellow Emperor's Classic), acupuncture has been used in China as a therapeutic measure for at least 2000 years. ${ }^{1}$ However, acupuncture as anaesthesia is a relatively recent development. It has a sound physiological and neurochemical basis. ${ }^{1}$ Although acupuncture anaesthesia is still widely used in China for craniotomy and thyroidectomy, it is not as frequently employed today for open heart operations in China as it was in the 1970s. The main reason is that the patients have to undergo lengthy preoperative training to practice slow and deep abdominal and diaphragmatic

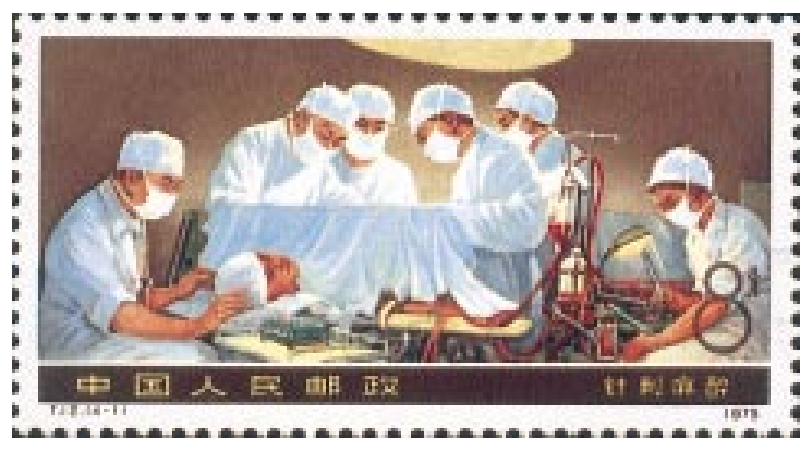

breathing required during the operation to overcome the positive atmospheric pressure in the event that the pleural cavity is inadvertently entered; another prerequisite is that the surgeons must be extremely gentle, meticulous, and speedy. Conventional anaesthesia obviates both of these requirements and is thus preferred by younger surgeons.

There is a phrase in Chinese shown on the stamp, 開心, which means "being happy". Literally translated, Kai Xin actually means to open the heart. This remarkable stamp certainly depicts such a happy moment in the history of Chinese cardiac surgery. To transform acupuncture from a form of traditional medicine for relief of pain to a form of anaesthesia for open heart surgery is truly a great leap forward.

TSUNG O CHENG

Professor of Medicine, The George Washington University Medical Center, Washington, DC 20037, USA

1 Han J-S. Physiologic and neurochemical basis of acupuncture analgesia. In: Cheng TO, ed. The international textbook of cardiology. New York: Pergamon Press, 1987:1124-32. 